\title{
INTERFERENCE OF SARS-COV-2 WITH THE HOMEOSTASIS OF VENTILATION AND PERFUSION IN THE LUNG
}

Preprint - May 10, 2020

\author{
Clemente F. Arias* \\ CIB, Centro de Investigaciones Biológicas Margarita Salas (CSIC), Madrid, Spain \\ GISC, Grupo Interdisciplinar de Sistemas Complejos, Madrid, Spain \\ Francisco J. Acosta \\ Departamento de Ecología, Universidad Complutense de Madrid, Spain \\ Federica Bertocchini \\ CIB, Centro de Investigaciones Biológicas Margarita Salas (CSIC), Madrid, Spain \\ Cristina Fernández-Arias \\ iMM, Instituto de Medicina Molecular, Lisboa, Portugal
}

\begin{abstract}
A growing number of studies suggest that SARS-CoV-2 could interfere with homeostatic mechanisms in the lung but the implications of this possible interference have not been fully explored in the literature. In this work, we examine the consequences that can be drawn from this hypothesis according to currently available knowledge. We suggest that one such consequence is the potential disruption of normal ventilation and perfusion of lung regions that may be distant from the infection sites. Loss of ventilation might result in local alveolar hypoxia and contribute to hypoxemia, which in turn could trigger homeostatic responses that enhance blood oxygenation by redistributing pulmonary blood circulation. Sudden changes in perfusion might then lead to the development of hydrostatic edema and eventually to vascular remodeling and inflammation. Therefore, the immune response might not be the only source of the substantial inflammation observed in lung tissues of patients with severe COVID-19, as is often assumed in the literature. The balance between the homeostatic and the immune reaction in each patient could account for the observed heterogeneity of the clinical manifestations of COVID-19.
\end{abstract}

*Correspondence clmntf@gmail.com

Keywords SARS-CoV-2 - COVID-19 $\cdot$ Homeostasis $\cdot$ Hypoxic pulmonary vasoconstriction $\cdot$ Renin-angiotensin system $\cdot$ Hydrostatic edema $\cdot$ Permeability edema

Abbreviations ACE2: angiotensin-converting enzyme 2; ARDS: acute respiratory distress syndrome; Ang II: angiotensin II; COVID-19: 2019 novel coronavirus disease; HAPE: high altitude pulmonary edema; HPV: hypoxic pulmonary vasoconstriction; RAS: renin-angiotensin system; SARS-CoV: severe acute respiratory syndrome coronavirus; SARS-CoV-2: severe acute respiratory syndrome novel coronavirus

\section{Introduction}

The novel coronavirus disease (COVID-19; previously known as 2019-nCoV), caused by the severe acute respiratory syndrome novel coronavirus (SARS-CoV-2) has spread worldwide since its initial report in Wuhan, China, in late 
December 2019. COVID-19 has a wide clinical spectrum, ranging from asymptomatic infection to severe viral pneumonia with respiratory failure and even death [1].

Most patients with severe COVID-19 exhibit signs of substantial inflammation, which may lead to lung tissue damage and accelerate the development of respiratory failure [2]. However, recent reports suggest that some of the clinical manifestations of respiratory failure in COVID-19 patients cannot be explained by the alveolar damage caused by the infection or the subsequent immune response, so other processes would be involved in the progression of the disease [3]. In this brief review, we suggest that these processes might be related to homeostatic mechanisms that control blood and air circulation in the lungs in healthy circumstances. We will argue that these physiological mechanisms might spread and amplify the effects of the infection, contributing to the combination of signs observed in patients with COVID- 19 .

\section{Interference of SARS-CoV-2 infection with the homeostasis of ventilation and perfusion in the lung}

SARS-CoV-2 and the related severe acute respiratory syndrome coronavirus (SARS-CoV) use the angiotensin-converting enzyme 2 (ACE2) to infect their target cells in the alveolar epithelium [4-6]. This enzyme plays a key role in the renin-angiotensin system (RAS), a hormonal system that regulates blood pressure in the organism and participates in blood volume homeostasis. Angiotensin II (Ang II) is the main effector agent of the RAS and other than being a potent vasoconstrictor, it is also an inductor of proliferation, inflammation, and fibrosis in a variety of tissues [7]. In physiological conditions, ACE2 inhibits these effects by degrading Ang II to produce Ang (1-7), which is an antagonist of Ang II [8].

The binding of SARS-CoV and SARS-CoV-2 to ACE2 has raised the hypothesis that they could somehow interfere with the function of the RAS in the lung $[4,5,9-12]$. In the process of infecting a pneumocyte, these viruses might foster the internalization and shedding of ACE2 from the surface of the cell, thereby repressing its enzymatic activity and indirectly increasing the levels of Ang II in lung tissues [5, 13, 14]. In this work, we will explore the potential consequences of the homeostatic hypothesis on the progression of the infections by SARS-CoV and SARS-CoV-2.

The expression of ACE2 in pneumocytes is circumscribed to the membrane surface in contact with the external environment [15]. Therefore, any viral interaction with the enzyme would take place in the alveolar space. This might lead to an unbalance in alveolar Ang II whose implications are difficult to judge owing to the limited available information about the physiological role of the RAS in the alveolus. In contrast, current knowledge suffices to conjecture the effects of an analogous viral interference with the RAS in the pulmonary interstitium. Several cell types, including pulmonary mast cells, smooth muscle cells, and fibroblasts also express ACE2 [16-18]. Even if these cells might not be infected by SARS-CoV and SARS-CoV-2 [19], the virus could still bind ACE2 present in their membranes and reduce its enzymatic activity. The subsequent rise in the local levels of interstitial Ang II would induce the contraction of smooth muscle cells that surround arterial vessels and lung airways via interaction with the angiotensin II receptor type 1 (ATR1) [9,20]. Therefore, viral interaction with ACE2 in the pulmonary interstitium would trigger the constriction of arterial vessels and lung airways located in the proximity of infected alveoli.

Viral-induced vaso- and bronchoconstriction would disrupt the ventilation/perfusion ratio in two different ways. In vasoconstricted arterioles, blood flow would be diverted towards upstream vessels offering lower resistance [21]. As for bronchoconstriction, it would entail a loss of ventilation in the alveolar units located downstream from the constricted segments [22]. Importantly, the presence of the virus at some point along the length of greater bronchial branches could suffice to induce its constriction and sharply reduce the ventilation of ample regions of the lung.

Pulmonary alveoli do not usually receive uniform ventilation and it is not exceptional for some of them to be deficiently oxygenated even in healthy conditions [21, 23]. In such poorly-ventilated areas of the lung, bronchoconstriction could further reduce airflow but given their already lower relative contribution to blood oxygenation, this effect would not necessarily imply a marked loss of pulmonary function. In contrast, the consequences of viral-induced bronchoconstriction could be very conspicuous in optimally aerated areas. In this case, it might curtail the airflow to respiratory units that were operating efficiently before the infection [24]. This could lead to the sudden appearance of hypoxic zones in otherwise well-ventilated regions of the lung, which could compromise their function.

In healthy conditions, whenever alveolar hypoxia exceeds a critical threshold, a physiological mechanism known as hypoxic pulmonary vasoconstriction (HPV) redirects the blood flow from areas with deficient ventilation to betteroxygenated sectors, optimizing systemic oxygen delivery [21, 25, 26]. It is natural to assume that alveolar hypoxia caused by viral-induced bronchoconstriction should trigger HPV as it does in other circumstances. Very short exposure to moderate hypoxia (of the order of seconds) suffices to onset HPV and if hypoxia does not reverse, significant increases in vascular pressure take place over the next 6 to 8 hours [25-27]. Therefore, the unbalance of the pulmonary 

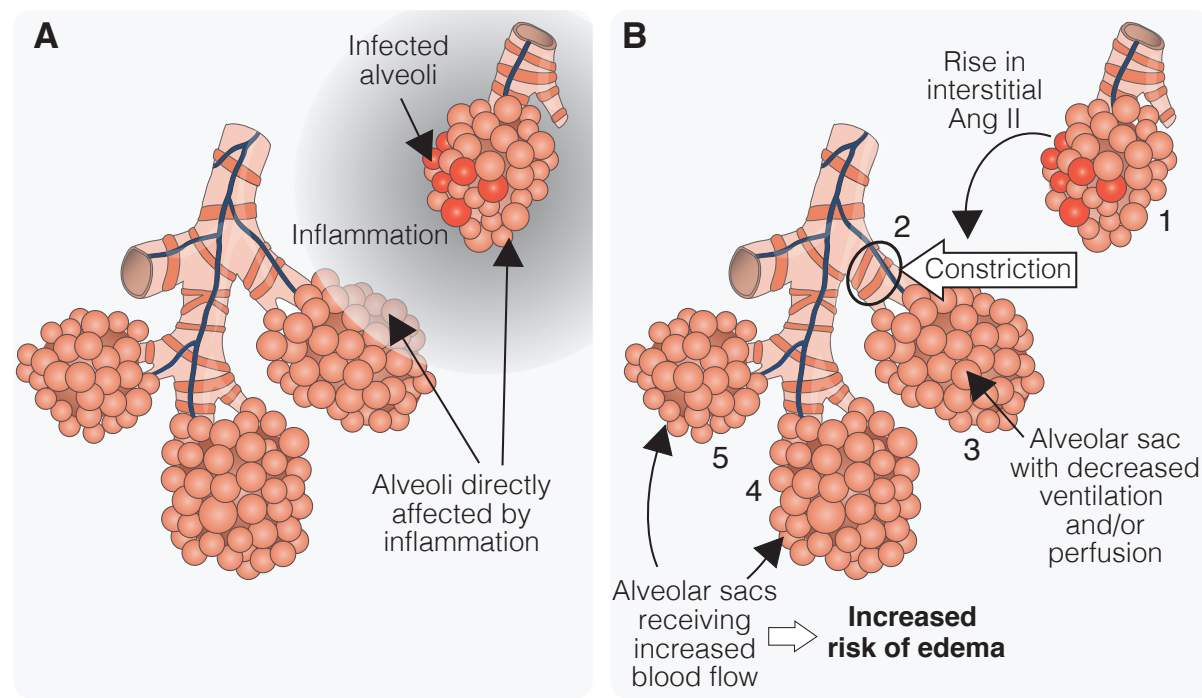

Figure 1: Spatial propagation of the RAS-mediated effect of SARS-CoV-2 infections. A) The inflammation caused by the immune reaction to the presence of the virus affects the immediate vicinity of the infective focus. B) In contrast, the respiratory units subject to the indirect effects of the viral-induced increased in interstitial Ang II could be relatively distant from the infection site. In the case illustrated in the figure, the infection of a few alveoli (1) could trigger vaso- and bronchoconstriction in blood vessels and lung airways present in surrounding interstitium (2), reducing perfusion and ventilation alveolar units that do not need to be connected with the infected ones (3). Blood flow diverted from these units would increase the perfusion of topologically connected alveolar sacs (4 and 5), increasing the risk of hydrostatic edema.

RAS caused by the presence of the virus would rapidly translate into a local redistribution of the pulmonary blood circulation, either directly by vasoconstriction or indirectly by bronchoconstriction-induced HPV.

\section{Two synergic pathways to inflammation in COVID-19}

What are the functional implications of the viral-induced changes in the pulmonary circulation? In order to address this issue, we begin by remarking that the alveolar units undergoing viral-induced vaso- and bronchoconstriction would not be necessarily infected by the virus (Fig. 11. The spatial extent of viral interference would depend on the location of constricted blood vessels and airways within the circulatory and ventilatory networks respectively. For instance, the constriction of a bronchiole would affect more respiratory units than the constriction of a single alveolar duct.

The effects of blood redistribution caused by viral-induced vasoconstriction and HPV would vary depending on the previous degree of constriction of the affected vascular vessels. Viral interference with the pulmonary RAS would be particularly adverse in those regions of the lung receiving greater blood flow. Small arterioles supplying blood to these regions would be largely unconstricted, so they would be at greater risk of fluid leakage in the case of sudden over-perfusion. In this situation, the extravascular accumulation of fluid might exceed the capacity of interstitial and alveolar fluid reabsorption, leading to hydrostatic edema and reduced gas exchange in the distressed respiratory units. In turn, edema might induce further changes in the local distribution of blood circulation [28, 29]. Therefore, in well-perfused areas, viral-induced vasoconstriction and HPV might trigger a positive feedback loop of circulatory rearrangements that might contribute to the spatial propagation of the indirect, RAS-mediated effects of the infection.

The progressive spread of viral-induced hydrostatic edema could eventually trigger a secondary inflammation reaction and lead to vascular remodeling and fibrosis [30], much as in other forms of hydrostatic edema [31, 32]. In poorly perfused regions, vascular vessels would be sufficiently constricted before the infection so as to accommodate unexpected rises in perfusion. For this reason, the risk of fluid leakage from small arterioles would be much lower and, consequently, the effects of the viral interferences with the pulmonary RAS would probably be negligible in this case.

From the previous remarks, it follows that those lung regions receiving higher ventilation and perfusion before the infection would be the most vulnerable to the effects of viral interference with the local pulmonary RAS. Furthermore, considering their substantial contribution to blood oxygenation relative to other areas, the RAS-mediated viral effects in these regions could be particularly deleterious. This impact might be even more pronounced in the elderly owing to the progressive reduction of ventilatory capacity with age [33]. Contrarily, viral interference with the pulmonary RAS in 


\begin{tabular}{|c|c|c|}
\hline & Low ventilation & High ventilation \\
\hline Low perfusion & No effects & Risk of hypoxia \\
\hline High perfusion & Risk of edema & Risk of edema and hypoxia \\
\hline
\end{tabular}

Table 1: Hypothesized effects of viral interference with the pulmonary RAS in lung regions depending on their ventilation and perfusion before the infection.

other lung areas would have less severe consequences (see Table 1). Therefore, the relative importance of viral-induced vasoconstriction and HPV in the progress of the disease would be determined by the particular spatial distribution of the infected lung areas in each patient.

The aspects of SARS-CoV-2 infections discussed so far are exclusively related to their potential interference with the pulmonary RAS leading to the disruption of the ventilation/perfusion ratio. However, it is obvious that this is not the only effect of the infection in the lung. Tissular stress caused by the replication of the virus in the infected cells and by the subsequent immune response leads to inflammation. This, in turn increases microvascular permeability and leukocyte infiltration and may cause permeability edema, with the subsequent impact on pulmonary function. The ensuing loss of ventilation in the affected alveoli could also trigger HPV and local redistribution of blood flow around the infective focuses, which would further boost the development of inflammatory edema [25, 33].

Respiratory failure from acute respiratory distress syndrome (ARDS) is currently the leading cause of mortality among COVID-19 patients [34] and in most severe cases, it is often accompanied by the cytokine release syndrome, which is a typical sign of hyperinflammation [35, 36]. It is often implicitly assumed in the literature that state is exclusively caused by the immune response to the virus. However, we have argued above that indirect, RAS-mediated effects of the viral infection could lead to hydrostatic edema in well-perfused regions of the lung and to the subsequent development of

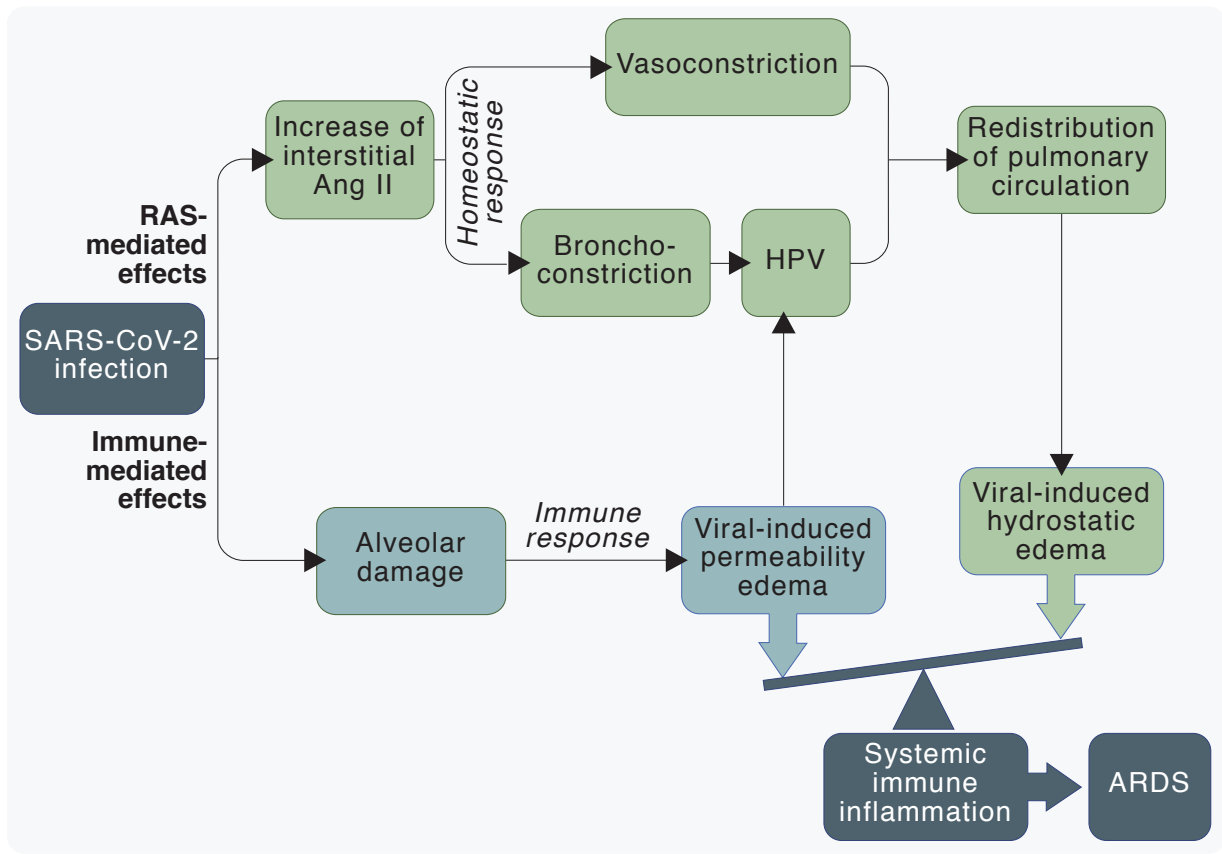

Figure 2: Two intertwined pathways to inflammation in SARS-CoV-2 infections. In this work, we suggest that the development of systemic immune inflammation and ARDS in the late stages of COVID-19 would result from the synergy of two simultaneous processes. The first one is the well-characterized immune inflammation triggered in response to the viral presence that may lead to the appearance of permeability edema. We suggest that the second one would naturally emerge from the interference of the virus with the pulmonary RAS that has been hypothesized in the literature. Viral-induced increase in interstitial Ang II would trigger the constriction of blood vessels and lung airways, which could result in alveolar hypoxia. Homeostatic mechanisms would then respond by locally redistributing the blood circulation. In some regions of the lung, this could cause hydrostatic edema and eventually lead to inflammation. The manifestations of the disease in its early stages would depend on the relative contribution of direct (immune-mediated) and indirect (RAS-mediated) effects of the virus. Differences among patients in the balance between both pathways would account for the observed interindividual heterogeneity in the manifestations of the disease. 
secondary inflammation. We suggest that this process could synergize with the immune-derived inflammation to drive the progress of the disease into a more damaging phase, eventually leading to the development of ARDS [4, 37.-39] (Fig. 2).

From this viewpoint, even if the late stages of the disease often converge to generalized systemic inflammation and ARDS, the particular pathway followed by the infection in its early phases could vary depending on the relative weight of immune-mediated and RAS-mediated viral effects in each patient. For instance, the infection of a few respiratory units located in particularly well-perfused and well-ventilated regions of the lung could lead to reduced gas exchange and hydrostatic edema accompanied by low levels of immune inflammation. In contrast, with higher levels of infection or more intense inflammatory responses, the immune-mediated effects would be more apparent. The symptoms arising from the synergy of both pathways could account for the atypical manifestations of ARDS in COVID-19 [40].

\section{Conclusions}

The consequences of viral-inflicted damage on lung tissues and the adverse effects that may arise from the ensuing immune response of the organism are well-known factors that account for some of the clinical signs of COVID-19. To the best of our knowledge, the implications of the viral interference with the pulmonary RAS hypothesized in recent studies [4, 5, 9-12] have been much less explored. In this work, we have argued that some of the manifestations of SARS-CoV-2 infections arise at least in part from the normal operation of homeostatic mechanisms in the abnormal context created by such viral interference with host homeostasis.

To begin with, in normal circumstances, increased pulmonary levels of Ang II usually respond to a variety of physiological signals that determine the optimal degree of constriction of blood vessels and lung airways. The interaction of the virus with ACE2 could be viewed as a source of misleading information in the pulmonary interstitium that would intensify constriction in the absence of appropriate physiological clues. The ensuing vaso- and bronchoconstriction would not respond to an actual need of the organism but to abnormal stimuli, which could have detrimental consequences. For instance, the anomalous constriction of airways in originally well-oxygenated areas of the lung could be a largely neglected factor in the development of hypoxemia and systemic hypoxia in some COVID-19 patients.

The viral disruption of the constriction of blood vessels and lung airways in the affected area would displace a number of respiratory units from their former equilibrium. In the case of alveolar hypoxia, the reduction in oxygen levels should trigger the same homeostatic compensatory mechanisms as in healthy circumstances. However, in the context of the infection, they could have undesirable consequences. For instance, HPV helps to maintain oxygen homeostasis in normal conditions by diverting blood flow away from areas with insufficient ventilation. In contrast, viral-induced HPV could reduce blood supply to lung areas that were optimally ventilated before the infection and increase the perfusion of poorly-ventilated regions [3]. It could also increase the risk of hydrostatic pulmonary edema in regions that were initially well perfused. Importantly, these homeostasis-related effects of the viral infection would no be circumscribed to the immediate surroundings of the infection sites.

Both the intensity of HPV in response to alveolar hypoxia and the susceptibility to develop HPV-mediated hydrostatic edema are highly variable among individuals [26, 41, 42]. On the other hand, the probability of viral-induced edema would also be modulated by other factors such as the ventilation/perfusion ratio of the pulmonary regions undergoing viral interference. The multifactorial susceptibility to the RAS-mediated effects of the infection could contribute to the observed heterogeneity in the clinical signs of COVID-19. In this regard, a recent study suggests that COVID-19 lung injuries could be similar to high altitude pulmonary edema (HAPE), a form of hydrostatic edema mediated by HPV that appears at high altitudes [43]. Other reports point in the opposite direction and affirm that there is no possible similarity between COVID-19 and HAPE [44].

In our opinion, the question that COVID-19 is not altitude sickness is beyond doubt. The ultimate cause of HAPE is global hypoxia owing to low atmospheric oxygen availability and it usually develops in the absence of a strong inflammatory response against a pulmonary infection. This is is in sharp contrast with the causes of COVID-19. Nevertheless, we have shown that a form of hydrostatic edema could arise as a potential consequence of viral-induced vasoconstriction and HPV in COVID-19. Those patients in which RAS-mediated effects were more prevalent than immune-mediated effects could exhibit signs of hydrostatic edema in the absence of apparent inflammation in the early stages of the disease, which could cause lung injury similar to HAPE. However, the clinical manifestations would be quite different for patients with dominant immune-mediated symptoms. The balance between RAS- and immune-mediated effects induced by the virus (see Fig. 2) could give rise to a continuum of pulmonary clinical signs between these two extremes, which could account for seemingly contradictory observations.

We want to stress that we are not advocating for the use of any particular clinical strategy over another. Instead, we intend to highlight an aspect of SARS-CoV-2 infections that might provide useful insight into the underlying causes of 
the clinical manifestations of COVID-19. Unfortunately, current quantitative knowledge about the RAS is insufficient to fully understand the effects that might derive from any attempt to offset viral-induced unbalance. Manipulating the levels of Ang II or trying to mitigate the RAS-mediated effects caused by the infection in the lung might lead to undesired consequences in other tissues or even in lung areas unaffected by viral interference.

SARS-CoV-2 might also interfere with the local RAS in other organs, such as the heart or the kidney. Furthermore, the infection often leads to hypoxia and hypoxemia, which must obviously disturb the homeostasis of oxygen and iron in the organism. We believe that deeper knowledge about how homeostatic mechanisms operate under healthy conditions would be necessary to understand the consequences of indirect, non-immune responses of the organism to infections. Many particular features of COVID-19 could well be impossible to understand without explicitly taking into account this homeostatic approach to the disease.

\section{References}

[1] Zhou, F. et al. Clinical course and risk factors for mortality of adult inpatients with COVID-19 in Wuhan, China: a retrospective cohort study. The Lancet 395, 1054-1062 (2020). URL https://linkinghub.elsevier . com/ retrieve/pii/S0140673620305663

[2] Cao, X. COVID-19: immunopathology and its implications for therapy. Nature Reviews Immunology 20, 269-270 (2020). URL https://doi.org/10.1038/s41577-020-0308-3

[3] Lang, M. et al. Hypoxaemia related to COVID-19: vascular and perfusion abnormalities on dual-energy CT. The Lancet Infectious Diseases S1473309920303674 (2020). URL https://linkinghub.elsevier.com/ retrieve/pii/S1473309920303674.

[4] Wu, Y. Compensation of ACE2 Function for Possible Clinical Management of 2019-nCoV-Induced Acute Lung Injury. Virologica Sinica (2020). URL https://doi.org/10.1007/s12250-020-00205-6.

[5] Kuba, K. et al. A crucial role of angiotensin converting enzyme 2 (ACE2) in SARS coronavirus-induced lung injury. Nature Medicine 11, 875-879 (2005). URL https ://www . nature .com/articles/nm1267

[6] Zhou, P. et al. A pneumonia outbreak associated with a new coronavirus of probable bat origin. Nature 579, 270-273 (2020). URL https://www.nature.com/articles/s41586-020-2012-7

[7] Benigni, A., Cassis, P. \& Remuzzi, G. Angiotensin II revisited: new roles in inflammation, immunology and aging. EMBO Molecular Medicine 2, 247-257 (2010). URL https://onlinelibrary.wiley.com/doi/abs/ 10.1002/emmm. 201000080

[8] Burrell, L. M., Johnston, C. I., Tikellis, C. \& Cooper, M. E. ACE2, a new regulator of the renin-angiotensin system. Trends in endocrinology and metabolism: TEM 15, 166-169 (2004). URL http://www.doi.org/10. 1016/j.tem.2004.03.001

[9] Wevers, B. A. \& van der Hoek, L. Renin-angiotensin system in human coronavirus pathogenesis. Future Virology 5, 145-161 (2010). URL https://www.ncbi.nlm.nih.gov/pmc/articles/PMC7079966/

[10] Verdecchia, P., Cavallini, C., Spanevello, A. \& Angeli, F. The pivotal link between ACE2 deficiency and SARSCoV-2 infection. European Journal of Internal Medicine (2020). URL https://www.ncbi.nlm.nih.gov/ pmc/articles/PMC7167588/

[11] Kai, H. \& Kai, M. Interactions of coronaviruses with ACE2, angiotensin II, and RAS inhibitors-lessons from available evidence and insights into COVID-19. Hypertension Research 1-7 (2020). URL https://www. nature. com/articles/s41440-020-0455-8.

[12] Imai, Y., Kuba, K. \& Penninger, J. M. The renin-angiotensin system in acute respiratory distress syndrome. Drug Discovery Today: Disease Mechanisms 3, 225-229 (2006). URL http://www.sciencedirect.com/ science/article/pii/S1740676506000472

[13] South, A. M., Tomlinson, L., Edmonston, D., Hiremath, S. \& Sparks, M. A. Controversies of renin-angiotensin system inhibition during the COVID-19 pandemic. Nature Reviews. Nephrology (2020). URL http://www . doi. org/10.1038/s41581-020-0279-4.

[14] Kuba, K., Imai, Y., Ohto-Nakanishi, T. \& Penninger, J. M. Trilogy of ACE2: a peptidase in the renin-angiotensin system, a SARS receptor, and a partner for amino acid transporters. Pharmacology \& Therapeutics 128, 119-128 (2010). URL http://www.doi.org/10.1016/j.pharmthera.2010.06.003

[15] Hamming, I. et al. Tissue distribution of ACE2 protein, the functional receptor for SARS coronavirus. A first step in understanding SARS pathogenesis. The Journal of Pathology 203, 631-637 (2004). URL https: //doi.org/10.1002/path.1570 
[16] Oarhe, C. I. et al. Hyperoxia downregulates angiotensin-converting enzyme-2 in human fetal lung fibroblasts. Pediatric Research 77, 656-662 (2015). URL https://doi.org/10.1038/pr.2015.27.

[17] Caughey, G. H., Raymond, W. W. \& Wolters, P. J. Angiotensin II generation by mast cell alpha- and beta-chymases. Biochimica Et Biophysica Acta 1480, 245-257 (2000). URL https://doi.org/10.1016/s0167-4838(00) 00076-5

[18] Qi, F., Qian, S., Zhang, S. \& Zhang, Z. Single cell RNA sequencing of 13 human tissues identify cell types and receptors of human coronaviruses. Biochemical and Biophysical Research Communications 526, 135-140 (2020). URL https://doi.org/10.1016/j.bbrc.2020.03.044

[19] Shieh, W.-J. et al. Immunohistochemical, in situ hybridization, and ultrastructural localization of SARS-associated coronavirus in lung of a fatal case of severe acute respiratory syndrome in Taiwan. Human Pathology 36, 303-309 (2005). URL https://linkinghub.elsevier.com/retrieve/pii/S0046817704006240

[20] Veerappan, A. et al. Mast cell renin and a local renin-angiotensin system in the airway: role in bronchoconstriction. Proceedings of the National Academy of Sciences of the United States of America 105, 1315-1320 (2008). URL https://doi.org/10.1073/pnas.0709739105

[21] Tarry, D. \& Powell, M. Hypoxic pulmonary vasoconstriction. BJA Education 17, 208-213 (2017). URL https://linkinghub.elsevier.com/retrieve/pii/S2058534917300471

[22] Kelly, V. J. et al. Hypoxic Pulmonary Vasoconstriction Does Not Explain All Regional Perfusion Redistribution in Asthma. American Journal of Respiratory and Critical Care Medicine 196, 834-844 (2017). URL https: //doi.org/10.1164/rccm.201612-24380C

[23] Lumb, A. B. \& Horner, D. Pulmonary Physiology. In Pharmacology and Physiology for Anesthesia, 445-457 (Elsevier, 2013). URL https://linkinghub.elsevier.com/retrieve/pii/B9781437716795000259

[24] Alagappan, V. K. T., de Boer, W. I., Misra, V. K., Mooi, W. J. \& Sharma, H. S. Angiogenesis and Vascular Remodeling in Chronic Airway Diseases. Cell Biochemistry and Biophysics 67, 219-234 (2013). URL https: //doi.org/10.1007/s12013-013-9713-6.

[25] Dunham-Snary, K. J. et al. Hypoxic Pulmonary Vasoconstriction. Chest 151, 181-192 (2017). URL https: //linkinghub.elsevier.com/retrieve/pii/S0012369216591624

[26] Swenson, E. R. Hypoxic Pulmonary Vasoconstriction. High Altitude Medicine \& Biology 14, 101-110 (2013). URL http://www.liebertpub.com/doi/10.1089/ham.2013.1010.

[27] Swenson, E. R. Hypoxic Pulmonary Vasoconstriction and Chronic Lung Disease. Advances in Pulmonary Hypertension 12, 135-144 (2013). URL http://advancesinph.org/doi/10.21693/1933-088X-12.3.135

[28] Mazzuca, E., Aliverti, A. \& Miserocchi, G. Understanding Vasomotion of Lung Microcirculation by In Vivo Imaging. Journal of Imaging 5, 22 (2019). URL https://www.mdpi . com/2313-433X/5/2/22

[29] Althaus, M., Clauss, W. G. \& Fronius, M. Amiloride-Sensitive Sodium Channels and Pulmonary Edema. Pulmonary Medicine 2011 (2011). URL https://www.ncbi.nlm.nih.gov/pmc/articles/PMC3100597/

[30] Ma, H., Xu, D., Wu, Y., Ma, Y. \& Li, Z. To decipher the hypoxic pulmonary hypertension: Vascular heterogeneity and the hypothesis of hypoxic responsive threshold. Journal of Medical Hypotheses and Ideas 9, 29-37 (2015). URL http://www.sciencedirect.com/science/article/pii/S2251729415000038.

[31] Swenson, E. R. \& Bärtsch, P. High-Altitude Pulmonary Edema (John Wiley \& Sons, Inc., Hoboken, NJ, USA, 2012). URL http://doi.wiley.com/10.1002/cphy.c100029

[32] Swenson, E. R. et al. Pathogenesis of High-Altitude Pulmonary Edema: Inflammation Is Not an Etiologic Factor. JAMA 287, 2228 (2002). URL http://jama.jamanetwork.com/article. aspx?doi=10.1001/jama. 287. 17.2228

[33] Hales, C. A. Physiological Function of Hypoxic Pulmonary Vasoconstriction. In Yuan, J. X. J. (ed.) Hypoxic Pulmonary Vasoconstriction: Cellular and Molecular Mechanisms, Developments in Cardiovascular Medicine, 3-14 (Springer US, Boston, MA, 2004). URL https ://doi.org/10.1007/1-4020-7858-7_1

[34] Mehta, P. et al. COVID-19: consider cytokine storm syndromes and immunosuppression. The Lancet 395, 1033-1034 (2020). URL https://linkinghub.elsevier.com/retrieve/pii/S0140673620306280.

[35] Ruan, Q., Yang, K., Wang, W., Jiang, L. \& Song, J. Clinical predictors of mortality due to COVID-19 based on an analysis of data of 150 patients from Wuhan, China. Intensive Care Medicine (2020). URL http: //link.springer.com/10.1007/s00134-020-05991-x 
[36] Zhang, C., Wu, Z., Li, J.-W., Zhao, H. \& Wang, G.-Q. The cytokine release syndrome (CRS) of severe COVID-19 and Interleukin-6 receptor (IL-6R) antagonist Tocilizumab may be the key to reduce the mortality. International Journal of Antimicrobial Agents 105954 (2020). URL https://linkinghub.elsevier.com/retrieve/pii/ S0924857920301047.

[37] Shi, Y. et al. COVID-19 infection: the perspectives on immune responses. Cell Death \& Differentiation 27, 1451-1454 (2020). URL http://www.nature.com/articles/s41418-020-0530-3.

[38] Wu, C. et al. Risk Factors Associated With Acute Respiratory Distress Syndrome and Death in Patients With Coronavirus Disease 2019 Pneumonia in Wuhan, China. JAMA Internal Medicine (2020). URL https:// jamanetwork.com/journals/jamainternalmedicine/fullarticle/2763184.

[39] Huang, C. et al. Clinical features of patients infected with 2019 novel coronavirus in Wuhan, China. The Lancet 395, 497-506 (2020). URL https://linkinghub.elsevier.com/retrieve/pii/S0140673620301835

[40] Gattinoni, L. et al. Covid-19 Does Not Lead to a “Typical” Acute Respiratory Distress Syndrome. American Journal of Respiratory and Critical Care Medicine rccm.202003-0817LE (2020). URL https ://www . ats journals org/doi/10.1164/rccm.202003-0817LE

[41] Frise, M. C. \& Robbins, P. A. Iron, oxygen, and the pulmonary circulation. Journal of Applied Physiology 119, 1421-1431 (2015). URL https://www.physiology.org/doi/10.1152/japplphysiol.00179.2015.

[42] Dawson, C. A. Hypoxic Pulmonary Vasoconstriction: Heterogeneity. Developments in Cardiovascular Medicine (Springer US, Boston, MA, 2004). URL https ://doi .org/10.1007/1-4020-7858-7_2.

[43] Solaimanzadeh, I. Acetazolamide, Nifedipine and Phosphodiesterase Inhibitors: Rationale for Their Utilization as Adjunctive Countermeasures in the Treatment of Coronavirus Disease 2019 (COVID-19). Cureus 12. URL https://www.ncbi.nlm.nih.gov/pmc/articles/PMC7096066/.

[44] Luks, A. M. et al. COVID-19 Lung Injury is Not High Altitude Pulmonary Edema. High Altitude Medicine \& Biology ham.2020.0055 (2020). URL https : //www.liebertpub.com/doi/10.1089/ham.2020.0055 